

163 | juillet-sptembre 2002

De la légende au mythe. Parole, langue et pensée

\section{Markos Zafiropoulos, Lacan et les sciences sociales. Le déclin du père (1938-1953)}

Paris, PUF, 257 p. (« Philosophie d'aujourd'hui »)

\section{Samuel Lézé}

\section{(2) OpenEdition}

\section{Journals}

Édition électronique

URL : http://journals.openedition.org/lhomme/12491

DOI : 10.4000//homme.12491

ISSN : 1953-8103

Éditeur

Éditions de l'EHESS

Édition imprimée

Date de publication : 21 juin 2002

Pagination : 250-251

ISBN : 2-7132-1771-7

ISSN : 0439-4216

\section{Référence électronique}

Samuel Lézé, «Markos Zafiropoulos, Lacan et les sciences sociales. Le déclin du père (1938-1953)»,

L'Homme [En ligne], 163 | juillet-sptembre 2002, mis en ligne le 10 juillet 2007, consulté le 24

septembre 2020. URL : http://journals.openedition.org/lhomme/12491 ; DOI : https://doi.org/10.4000/ Ihomme.12491

Ce document a été généré automatiquement le 24 septembre 2020

(c) École des hautes études en sciences sociales 


\section{Markos Zafiropoulos, Lacan et les sciences sociales. Le déclin du père (1938-1953)}

Paris, PUF, 257 p. (« Philosophie d'aujourd'hui »)

\section{Samuel Lézé}

1 LA LITTÉRATURE psychanalytique laisse l'impression de ne cesser d'accumuler lectures et relectures. La clinique même est lecture d'un inconscient confrontée à la relecture des textes classiques qui en donneraient la clé. Sans doute la psychanalyse est-elle sortie complète de la tête de Freud. Dans son dernier ouvrage, Markos Zafiropoulos, sociologue et psychanalyste, constate et déplore l'autoréférentialité du champ analytique plus qu'il ne l'explique : il ne poursuit pas sur cette piste que commencent à aborder quelques ethnologues. L'auteur ne déroge donc pas aux lois du genre puisqu'il propose une lecture, jusqu'alors négligée, de l'œuvre de Lacan dans son rapport aux sciences sociales.

2 À travers une lecture des lectures du jeune Lacan, l'auteur tente d'identifier dans un corpus de textes allant de 1938 à 1953 les emprunts de Jacques Lacan aux sciences sociales lorsqu'il n'était pas encore tout à fait freudien. Il découvre ainsi un «Lacan durkheimien" antérieur au "Lacan lévi-straussien» qui fera l'objet d'un prochain ouvrage. Cette découverte aurait bien peu d'intérêt si elle ne permettait de forcer certains problèmes à se déclarer : l'œEdipe et ses conditions sociales, ou « les conditions sociales de l'œdipisme ", selon une formulation inattendue de Lacan ${ }^{1}$.

3 Zafiropoulos prête tout d'abord attention au texte sur les "complexes familiaux" (1938) traitant du processus de maturation subjectif qui introduit en psychanalyse la thèse du déclin de la famille et du père (chap. I), texte qu'il commente en détail afin de la confronter aux thèses durkheimiennes sur la famille (la loi de contraction familiale; chap. II) et à ses sources : la dévaluation du père provient de la «mise en conjugalité " (p.62). D'où la théorie de l'anomie sociale et de l'émergence de l'individualisme. L'auteur dénonce cette thèse, toujours active selon lui dans le champ psychanalytique en tant qu'« illusion patriarcale ». 
4 Cette thèse implique par ailleurs une sociogenèse conjointe des névroses et de la psychanalyse. Il s'agit donc d'une conception des névroses (et de l'œedipe) qui est à relier à une déficience réelle du père de famille. Il s'agit aussi d'une conception fonctionnaliste de la psychanalyse qui trouverait son origine dans les besoins contemporains de soin.

5 Il en va de même de la conception socioculturelle du surmoi que défend Lacan en 1950 (chap. III): les psychopathes souffriraient d'un défaut de cette instance de normalisation sociale, instance dans laquelle il faudrait chercher la solution de l'énigme du passage de la nature à la culture. C'est à la même époque que Lacan fait la découverte du symbolique tel que Lévi-Strauss le thématise dans son fameux commentaire de l'œuvre de Marcel Mauss.

6 À partir de nombreuses recherches de sciences sociales, Zafiropoulos montre aisément que la thèse du déclin de la fonction paternelle n'est pas historiquement démontrée (chap. IV) et en tire les conséquences attendues (chap.V): la prolifération des conduites anomiques, dont le suicide est l'exemple durkheimien par excellence, ne doit rien aux défaillances de la famille réelle et concrète ; Durkheim est sous l'emprise d'une idéalisation de la famille. Il en va surtout ainsi de l'œedipe, dont l'auteur, bien entendu, une fois écarté le problème des conditions sociales, présuppose l'existence sans en accroître l'intelligibilité ni en discuter la validité. Il a néanmoins le mérite de relancer la recherche sur les conditions de la formation du champ analytique.

7 En retraçant les emprunts malheureux (le sociologisme de Durkheim et sa thèse du déclin du père) et heureux (le symbolique de Mauss et de Lévi-Strauss) de Lacan aux sciences sociales, Zafiropoulos dégage d'une part la genèse de la théorie du nom du père (la loi) - fonction symbolique autonome, indépendante des membres de la famille - qui distingue les registres du Réel, du Symbolique et de l'Imaginaire (1953), et dont se déduit le sujet de l'inconscient. Il montre d'autre part comment Lacan devient enfin freudien en dégageant la psychanalyse d'une sociogenèse inacceptable, réaccentuant ainsi son autonomie - la portant même à sa limite extrême. Désormais, s'il faut revenir à Freud, c'est pour comprendre les conditions de l'autoréférentialité du champ analytique.

\section{NOTES}

1. Écrits, Paris, Seuil, 1966 (« Le champ freudien ») : 136. 


\section{AUTEUR}

\section{SAMUEL LÉZÉ}

ENS, Laboratoire de sciences sociales, Paris. 